[Chem. Pharm. Bull.

35( 6 ) 2495-2503(1987) $]$

\title{
Separation and Identification of Amine-Carbohydrate Reaction Product in Aqueous Solution
}

\author{
Satoshi OKada, ${ }^{* a}$ Hiroshi Nakahara,${ }^{a}$ Hiroshi Isaka, ${ }^{a}$ \\ TOORU TAGA, ${ }^{b}$ and KOICHIRO MIYAJIMA ${ }^{b}$ \\ National Institute of Hygienic Sciences, Osaka Branch, ${ }^{a}$ 1-1-43, Hoenzaka, \\ Higashi-ku, Osaka 540, Japan and Faculty of Pharmaceutical Sciences,

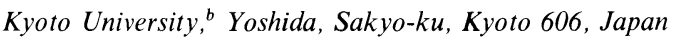

(Received October 17, 1986)

\begin{abstract}
Drugs with a primary aromatic amino moiety such as procaine, procainamide, and $p$ aminobenzoic acid (PABA) are capable of reacting with various reducing sugars containing hexoaldoses and pentoaldoses. The amine-carbohydrate reaction products can be separated from the parent drugs by high performance liquid chromatography (HPLC) analysis. The formation and dissociation profiles of the products were followed by HPLC and ultraviolet spectroscopy. The reaction products formed in aqueous solution were not association complexes, but chemical reaction products, which were identified as glycosylamines or $\mathrm{N}$-glycosides, by comparison with authentic samples obtained by the condensation of amines with carbohydrates in methanol. An Xray analysis of one of the glycosylamines, 2-deoxy- $N$ - $p$-carboxyphenyl-D-ribosylamine, was carried out, and the absolute conformation and configuration in the crystalline state were determined.
\end{abstract}

Keywords_- $p$-aminobenzoic acid (PABA); procaine; procainamide; glucose; 2-deoxyribose; amine-carbohydrate condensate; glycosylamine; HPLC analysis; UV spectroscopy; X-ray analysis

In the pharmaceutical field, a number of carbohydrates have been widely used as diluent bases (expanders), binders, disintegrators, taste-modifying agents, and so on. Moreover in many parenteral solutions, various kinds of carbohydrates are prescribed as a main component, and many other drugs are often dissolved in such parenteral solutions for clinical use. Thus, numerous studies on the interaction of drugs with carbohydrates have been done. Over thirty years ago, from the anomalous changes in optical rotation of glucose solutions containing procaine, Cannell suggested that condensation occurred to form $N$-(Dglucosyl)procaine. ${ }^{1)}$ Later, $\mathrm{Kaito}^{2)}$ and $\mathrm{Ikeda}^{3)}$ also reported the $\mathrm{N}$-glucoside formation of procaine in aqueous glucose solutions. In these studies the reaction was followed by polarimetry, ${ }^{1)}$ by paper chromatography, ${ }^{2)}$ and by chemical analysis of the parent compound after solvent extraction. ${ }^{3)}$ Recently, the interaction of drugs with carbohydrates has been examined again, with the use of modern analytical methods. ${ }^{4)}$ Kirschenbaum et al. observed a decrease of the procainamide concentration in $5 \%$ dextrose solution to less than $90 \%$ of the original concentration after $24 \mathrm{~h}$ of storage. ${ }^{5}$ Baaske et al. reported similar phenomena and suggested the direct reaction of glucose with procainamide to yield one or more entirely new compounds. ${ }^{6)}$ Thereafter, Das Gupta examined the procainamide-glucose reaction product by high performance liquid chromatography (HPLC) analysis and described this reaction as complex formation. ${ }^{7)}$

In a study of the effect of carbohydrate on the stability of local anesthetics, we also found new reaction products of procaine, procainamide, and $p$-aminobenzoic acid (PABA) with several reducing sugars by HPLC analysis. The amine-carbohydrate reaction products separated chromatographically from the parent drugs were expected to be glycosylamines or 
$N$-glycosides, ${ }^{8)}$ as reported in the previous studies. ${ }^{1-3)}$ The present study was performed in order to determine whether the new reaction products are glycosylamines or not.

\section{Experimental}

Materials - Procaine (PC) was supplied by Fuso Pharmaceutical Industries Co., Ltd., and procainamide by Midori-Juji Co., Ltd. PABA was purchased from Wako Pure Chemical Industries Co., Ltd., and recrystallized from methanol-water several times. D-Glucose was provided by Meito Industries Co., Ltd., and other carbohydrates (Dgalactose, D-ribose, and 2-deoxy-D-ribose) were purchased from Nakarai Chemical Co., Ltd. Deionized distilled water and acetonitrile of chromatographic grade were used for the preparation of the eluent for HPLC analysis. Methanol was of chromatographic grade and other chemicals were of reagent grade (used without further purification).

Preparation of $N$-p-Carboxyphenyl-D-glycosylamines ( $N$-PABA-GA) $~ N$-PABA-GA were prepared by condensation of PABA with D-glucose, D-galactose, D-ribose, and 2-deoxy-D-ribose (dRib). They were obtained by reacting PABA with equimolar amounts of the above carbohydrates suspended in methanol for $0.5-3 \mathrm{~h}$ at $20-60^{\circ} \mathrm{C}$ until the attainment of complete miscibility. On cooling and/or evaporation of the solvent under reduced pressure at below $40^{\circ} \mathrm{C}$, solids or crystals were separated. They were crystallized from methanol twice or three times, and dried in vacuo at $40-60^{\circ} \mathrm{C}$. In the case of 2-deoxy- $N$-p-carboxyphenyl-D-ribosylamine ( $N$-PABA-dRib), large single crystals were obtained, and subjected to X-ray analysis. The physical properties of several $N$-PABA-GAs are summarized in Table I. Elementary analysis of $N$-PABA-dRib did not give complete agreement of the experimental values with the calculated ones due to the presence of residual methanol of solvation (Table I). Moreover the $N$-PABA-GAs were not stable, and turned brown during storage at room temperature for several months. Eventually they decomposed completely to black, tarry substances, as reported by Ellis and Honeyman. ${ }^{91}$

Formation and Dissociation of Amine-Carbohydrate Reaction Product-Formation of the Reaction Product: Reaction of an amine with a carbohydrate was initiated by mixing them in phosphate buffer $(0.1 \mathrm{M}, \mathrm{pH} 7.0$, ionic strength 0.3 ), in a $10 \mathrm{ml}$ centrifuge tube with a stopper. Initial concentrations of amine and carbohydrate were $0.5-$ $2.0 \mathrm{~mm}$ and $0.05-0.25 \mathrm{M}$, respectively. The reaction mixture was kept at $40 \pm 0.1{ }^{\circ} \mathrm{C}$ in a water bath under thermostatic control. Aliquots of $5-10 \mu \mathrm{l}$ of the solution were taken at appropriate intervals, and subjected to HPLC analysis.

Dissociation of the Reaction Product: The amine-carbohydrate reaction is a reversible equilibrium system, and the equilibrium was easily disturbed by simple dilution. After $2.5-10$ fold dilution of the equilibrated mixture $(2 \mathrm{~mm}$ amine $+0.25 \mathrm{M}$ carbohydrate), dissociation or degradation of the reaction product was followed by HPLC analysis. The diluting solution was the same buffer solution as that used in the formation procedure. The diluted samples were kept at $40 \pm 0.1{ }^{\circ} \mathrm{C}$, and at appropriate intervals the changes of the composition were followed by HPLC. In order to follow the dissociation process by spectroscopy, $40-50$ fold dilution of the equilibrated mixture was necessary. The spectral measurements were carried out under ambient conditions.

For examination of the hydrolysis of authentic glycosylamine, a definite amount of glycosylamine dissolved in methanol $(3.93 \mathrm{~mm})$ was mixed with a buffer solution of the desired $\mathrm{pH}$ value, and the hydrolytic behavior was

TABLE I. Physical Data for $N$ - $p$-Carboxyphenyl-D-glycosylamines ( $N$-PABA-GA)

\begin{tabular}{|c|c|c|c|c|c|c|c|}
\hline \multirow{2}{*}{ Glycosylamine } & \multirow{2}{*}{$\begin{array}{l}\text { Mol. formula } \\
\qquad\left(M_{\mathrm{r}}\right)\end{array}$} & \multirow{2}{*}{$\begin{array}{l}\mathrm{mp} \\
\left({ }^{\circ} \mathrm{C}\right)\end{array}$} & \multirow{2}{*}{$\begin{array}{l}{[\alpha]_{\mathrm{D}}^{20}} \\
\left({ }^{\circ}\right)^{a)}\end{array}$} & \multirow{2}{*}{$\begin{array}{c}\lambda_{\max } \\
(\mathrm{nm})^{b)}\end{array}$} & \multicolumn{3}{|c|}{$\begin{array}{l}\text { Analysis (\%) } \\
\text { Found (Calcd) }\end{array}$} \\
\hline & & & & & $\mathrm{C}$ & $\mathrm{H}$ & $\mathbf{N}$ \\
\hline$N$-p-Carboxyphenyl-D-glucosylamine & $\begin{array}{c}\mathrm{C}_{13} \mathrm{H}_{19} \mathrm{NO}_{7} \\
(301.29)\end{array}$ & $\begin{array}{l}165-170 \\
\text { (dec.) }\end{array}$ & -77 & 270 & $\begin{array}{r}52.3 \\
(52.2\end{array}$ & $\begin{array}{l}5.9 \\
5.7\end{array}$ & $\begin{array}{l}4.7 \\
4.7)\end{array}$ \\
\hline$N$-p-Carboxyphenyl-D-galactosylamine & $\begin{array}{c}\mathrm{C}_{13} \mathrm{H}_{19} \mathrm{NO}_{7} \\
(301.29)\end{array}$ & $\begin{array}{l}150-155 \\
\text { (dec.) }\end{array}$ & -112 & 271 & $\begin{array}{r}52.0 \\
(52.2\end{array}$ & $\begin{array}{l}5.7 \\
5.7\end{array}$ & $\begin{array}{l}4.6 \\
4.7)\end{array}$ \\
\hline 2-Deoxy- $N$ - $p$-carboxyphenyl-D-ribosylamine & $\begin{array}{c}\mathrm{C}_{12} \mathrm{H}_{15} \mathrm{NO}_{5} \\
(255.24)\end{array}$ & $\begin{array}{l}140-145 \\
\text { (dec.) }\end{array}$ & +20 & 271 & $\begin{array}{r}55.9 \\
(56.9\end{array}$ & $\begin{array}{l}6.4 \\
6.0\end{array}$ & $\begin{array}{l}5.3^{c} \\
5.5)\end{array}$ \\
\hline$N$-p-Carboxyphenyl-D-ribosylamine & $\begin{array}{c}\mathrm{C}_{12} \mathrm{H}_{15} \mathrm{NO}_{6} \\
(269.24)\end{array}$ & $\begin{array}{l}147-151 \\
\text { (dec.) }\end{array}$ & +67 & 270 & & - & \\
\hline
\end{tabular}

a) Optical rotation was measured in methanol and extrapolated to zero time. b) UV absorption was measured in phosphate buffer solution ( $\mathrm{pH} 7.0$ ) immediately after dissolution. c) The disagreement may have arisen from residual methanol of solvation. If $1 / 2 \mathrm{MeOH}$ remains in one 2-deoxy- $N$ - $p$-carboxyphenyl-D-ribosylamine molecule, the experimental values would agree well with the calculated ones $(\mathrm{C}: \mathrm{H}: \mathrm{N}=55.8: 6.3: 5.2)$. 


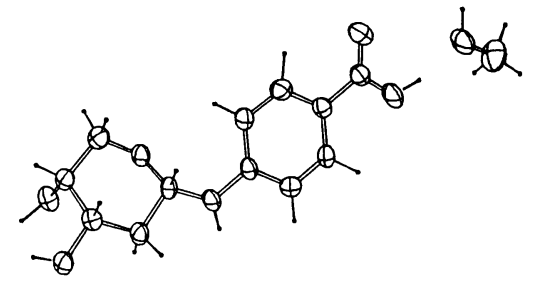<smiles>COCCOC</smiles>

(a)

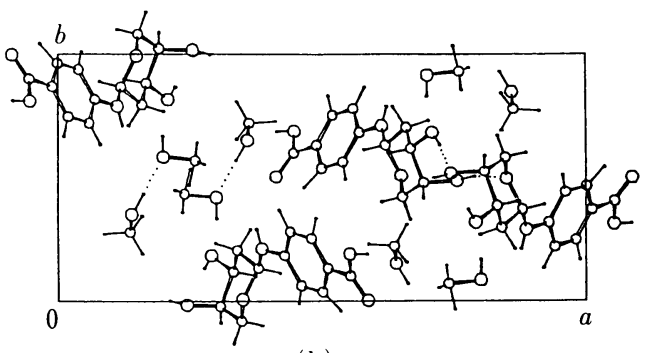

(b)
Fig. 1. X-Ray Analysis of 2-Deoxy- $N$-p-carboxyphenyl-D-ribosylamine ( $N$-PABA-dRib)

a) Perspective view of the $N$-PABA-dRib molecule with two molecules of methanol of solvation.

b) Projection along the $c$-axis.

followed by HPLC and ultraviolet (UV) spectroscopy as described above.

HPLC Analysis - An HPLC apparatus (model LC-5A) equipped with a UV detector (model SPD-2A) and with a photodiode array detector (model SPD-MIA) (Shimadzu Co.) was used, and the wavelength was set at 270 or $280 \mathrm{~nm}$. The stationary phase used was CHEMCOSORB 7 DPH $(15 \mathrm{~cm}$ long $\times 4.6 \mathrm{~mm}$ i.d., Chemco Co. $)$, and the mobile phase consisted of acetonitrile-sodium acetate buffer $(50 \mathrm{~mm}, \mathrm{pH} 3.5)$ which was varied in composition from $97: 3$ to $84: 16$ according to the combination of amine and carbohydrate. The flow rate was $1.5 \mathrm{or} 1.8 \mathrm{ml} / \mathrm{min}$. A short column ( $3 \mathrm{~cm}$ long $\times 4.6 \mathrm{~mm}$ i.d.) packed with CHEMCOSORB $7 \mathrm{DPH}$ was used to guard the main column. All chromatographic operations were carried out under ambient conditions.

Physical Measurements - Melting points were determined on a micromelting point apparatus (Yanagimoto Co.). UV absorption spectra were measured with model 100-60 and model 556 spectrophotometers (Hitachi Co.) under ambient conditions. Optical rotations of authentic $N$-PABA-GA in methanol were followed by with a model PM-201 polarimeter (Union Co.) at $20 \pm 0.1^{\circ} \mathrm{C}$, using a $10 \mathrm{~cm}$ cell with a stopper.

X-Ray Analysis of $N$-PABA-dRib - Single crystals of $N$-PABA-dRib were prepared by slow crystallization from methanol. Since the crystals were not stable and lost transparency rapidly on filtration, a crystal was sealed in a glass capillary in the solvent of recrystallization (methanol) for X-ray analysis. The crystal was orthorhombic, space group $P 22_{1} 22_{1}$ with the unit cell dimensions $a=21.490(4), b=9.999(2), c=7.448(2) \AA$, and $D_{\text {calcd }}=1.317 \mathrm{~g} \cdot \mathrm{cm}^{-3}$ for $Z=4\left(\mathrm{C}_{14} \mathrm{H}_{23} \mathrm{NO}_{7}\right.$ or $\left.\mathrm{C}_{12} \mathrm{H}_{15} \mathrm{NO}_{5} \cdot 2\left(\mathrm{CH}_{3} \mathrm{OH}\right), M_{\mathrm{r}} 317.34\right)$; eight solvent (methanol) molecules were included in a unit cell. A crystal with dimensions of $0.5 \times 0.4 \times 0.2 \mathrm{~mm}$ was used for data collection. The intensity data were measured on a Rigaku AFC-5RU diffractometer with graphite-monochromated $\mathrm{Cu} K_{\alpha}$ radiation $(\lambda=1.54178 \AA)$, using the $\omega-2 \theta$ scan method at the rate of $16^{\circ} \cdot \mathrm{min}^{-1}$. The structure was solved by the direct method using MULTAN 78, and refined by the full-matrix least-squares method. The final $R$-value was 0.057 for 1365 observed reflections. All computations were performed on a FACOM M382 in the Data Processing Center of Kyoto University, using the KPPXRAY programs.

As shown in Fig. 1, the 2-deoxy-D-ribose sugar ring has a pyranose chair form in the ${ }^{1} \mathrm{C}_{4}$ conformation, and the anomeric carbon atom has the $\beta$-configuration. Thus this compound exists as 2 -deoxy- $N$ - $p$-carboxyphenyl- $\beta$-Dribopyranosylamine in the crystalline state.

\section{Results and Discussion}

\section{Separation of Amine-Carbohydrate Reaction Product}

It is well-known that PC is easily hydrolyzed to PABA and diethylaminoethanol in weakly alkaline solutions. The hydrolysis can also occur in aqueous carbohydrate solutions. 
After partial hydrolysis of PC in $0.25 \mathrm{M}$ dRib solutions, an aliquot was subjected to HPLC analysis. A typical chromatogram is shown in Fig. 2. Five peaks were detected in the following order of separation: PABA-dRib reaction product, PABA, PC-dRib reaction product, PC, and $p$-nitrophenol (internal standard).

Retention times of the reaction products were shorter than those of the parent drugs, due to higher hydrophilicity. Although Das Gupta failed to detect the PABA-glucose reaction product, ${ }^{7)}$ we found the product and also PABA-dRib reaction product, as described below. Like PC and PABA, procainamide could also react with $\mathrm{dRib}$ and glucose. Thus the aminecarbohydrate reaction product detected in HPLC analysis seems not to be specific to the combination of procainamide and glucose as reported by Das Gupta ${ }^{7 a)}$ and others. ${ }^{5-6)}$ From previous data on the formation of $N$-(glycosyl)procaine with reducing sugars, ${ }^{1-3)}$ the reaction products found in the present study were suggested to be the condensation products of amines with carbohydrates.

\section{Formation and Dissociation of the Reaction Product}

In Fig. 3 the mode of formation of the reaction product between PABA and $\mathrm{dRib}$ is shown in terms of successive chromatograms after the addition of PABA to $0.25 \mathrm{M} \mathrm{dRib}$

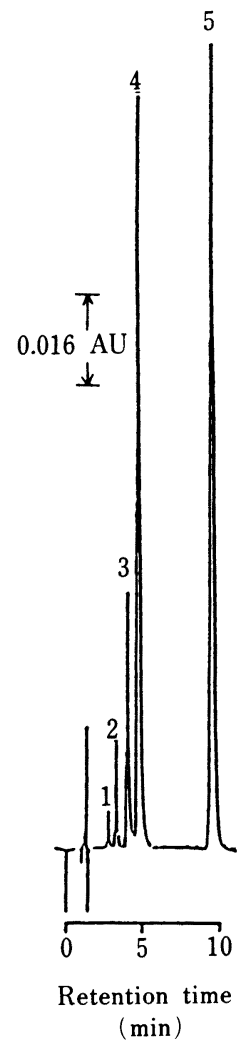

Fig. 2. Chromatographic Separation of the Amine-Carbohydrate Reaction Product

Peaks $1-5$ denote PABA-dRib reaction product, PABA, PC-dRib reaction product, $\mathrm{PC}$ and $p$-nitrophenol (internal standard), respectively.

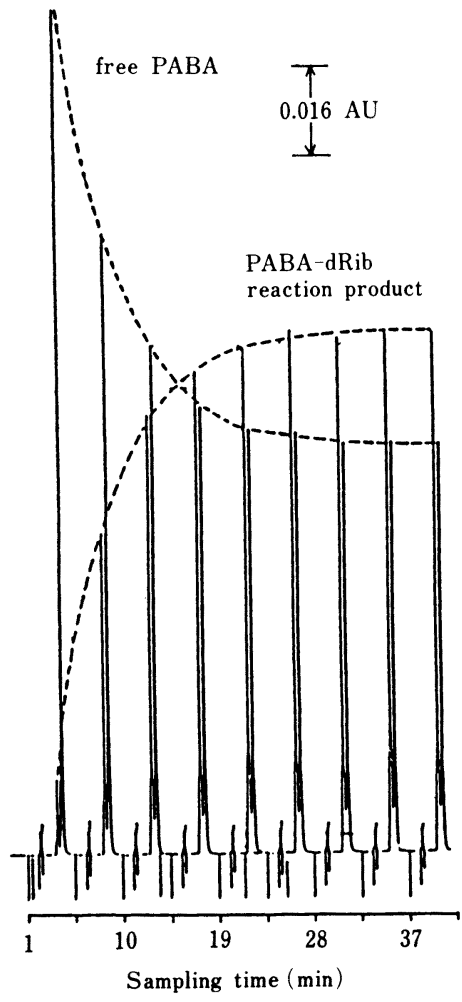

Fig. 3. Successive Chromatograms of the PABA-dRib System after Mixing of the Components at $\mathrm{pH} 7.0$ and at $40^{\circ} \mathrm{C}$

The reaction mixture was composed of PABA (1.83 $\mathrm{mM})$, dRib (250 mM), and phosphate buffer ( $100 \mathrm{mM}$, $\mathrm{pH}$ 7.0). The descending and ascending dotted lines, which connect the elution peaks, indicate the changes of the relative amounts of free PABA and PABA $\mathrm{dRib}$ reaction product in the medium, respectively. 


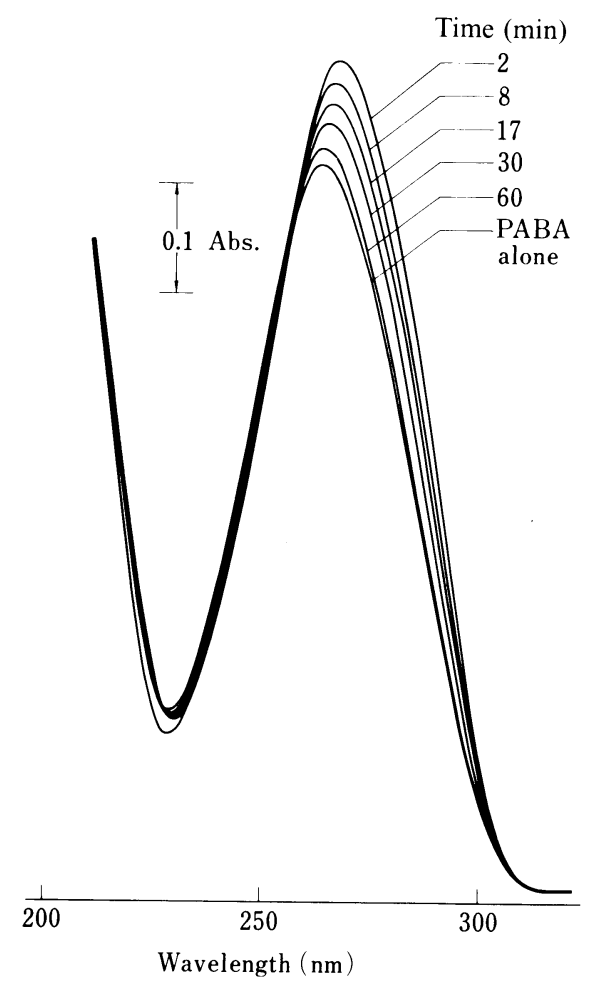

Fig. 4. UV Spectral Changes Due to the Dissociation of PABA-dRib Reaction Product at pH 7.0

UV spectral changes of PABA-dRib system were recorded after 40 -fold dilution of the equilibrated PABA-dRib mixture (1.83 mM PABA, 0.2 M dRib, $0.1 \mathrm{M}$ phosphate buffer, $\mathrm{pH} 7.0$ ).

solutions $\left(\mathrm{pH} 7.0,40^{\circ} \mathrm{C}\right)$. A rapid increase of the reaction product occurred concomitantly with a decrease of free PABA, as indicated by the dotted lines. The reaction had almost reached equilibrium after 30 min under the present conditions. The peak height ratio of the product to free PABA at equilibrium changed linearly with the concentration of $\mathrm{dRib}$ at a constant concentration of PABA. That is, the ratio increased with increment of the relative concentration of the carbohydrate.

On the other hand, when the equilibrium mixture of PABA-dRib was diluted with buffer solutions (same components as the reaction medium), a rapid dissociation of the product and establishment of a new equilibrium were observed by HPLC. Furthermore the process of dissociation could be followed by UV spectroscopy, as shown in Fig. 4. The PABA-dRib reaction product gradually dissociated into free PABA and dRib after 40 -fold dilution at room temperature. After $60 \mathrm{~min}$, the dissociation was almost complete and nearly the same spectrum as that of an equimolar concentration of standard PABA solution $\left(4.57 \times 10^{-5} \mathrm{M}\right)$ was obtained. Thus, in highly diluted PABA-dRib mixtures only a small amount of the reaction product remains. These spectral changes showed two characteristic features: one is the hypsochromic shift of the absorption maxima by about $4-5 \mathrm{~nm}$ in the course of the dissociation, and the other is the existence of the isosbestic point at around $255 \mathrm{~nm}$.

When a large excess of $\mathrm{dRib}$ exists compared to PABA, formation and dissociation of the product can be treated as a reversible 1st-order reaction; these results will be reported elsewhere. Reaction of PABA with glucose also occurred under the same conditions in the case of the PABA-dRib system, but the rate was much slower.

The above-mentioned chromatographic and spectroscopic behavior of amine-carbohydrate mixtures might be interpreted in terms of complex formation, as suggested in some recent reports. ${ }^{5-7)}$ However, in general, complex formation based on weak intermolecular forces is a rapid process in homogeneous systems under usual conditions. Thus, many 
organic complexes cannot be separated from their solutions as definite compounds, and they are often difficult to detect by usual chemical and physical means. ${ }^{10)}$ In contrast, the present amine-carbohydrate interaction proceeds slowly (Figs. 3 and 4), and in the case of PABA-glucose mixture the reaction rate is much slower than in the PABA-dRib system under the same conditions, as mentioned above. Moreover, the rate of the reaction is markedly dependent on temperature, as also found by Baaske et al. ${ }^{6)}$ and Ikeda, ${ }^{3)}$ which suggests the interaction to be a chemical reaction with a fairly large activation energy. Thus, it is reasonable to consider the present amine-carbohydrate interaction as being not a complex formation, but a reversible chemical reaction, like the mutarotation of reducing sugars. ${ }^{11}$ )

\section{Identification of Amine-Carbohydrate Reaction Products}

If, as discussed above, the products are chemical reaction products of amines and carbohydrates, such as glycosylamines or $\mathrm{N}$-glycosides,${ }^{8)}$ their formation corresponds to the condensation of amines with carbohydrates, and their dissociation, to the hydrolysis of glycosylamines. Several kinds of $N$-PABA-GAs were synthesized by condensation of PABA with various reducing sugars in methanol (see Table I). Confirmation of the structure of $\mathrm{N}$ PABA-dRib was obtained by X-ray analysis (see Fig. 1 and Experimental). As can be seen in Fig. 1, a covalent bond is formed between the anomeric carbon of $\mathrm{dRib}$ and the amino nitrogen of PABA, and the interatomic distance of the $\mathrm{C}-\mathrm{N}$ single bond is estimated to be $1.437 \AA$, as compared to the calculated value of $1.47 \AA .^{12)}$ Thus, at least in methanol, the primary aromatic amine and reducing sugar molecules are covalently combined with elimination of water, resulting in the formation of the glycosylamine.

A typical condensation reaction with elimination of water is observed in the reaction of aldehydes with primary amines,

$$
\mathrm{RCHO}+\mathrm{R}^{\prime} \mathrm{NH}_{2} \rightleftharpoons\left[\begin{array}{c}
\mathrm{OH} \\
\mathrm{I} \\
\mathrm{R}-\mathrm{C}-\mathrm{H} \\
1 \\
\mathrm{NHR}^{\prime}
\end{array}\right] \rightleftharpoons \mathrm{RCH}=\mathrm{NR}^{\prime}+\mathrm{H}_{2} \mathrm{O}
$$

where the intermediates and the resulting products are called carbinolamines and imines or Schiff bases, respectively. However, most imines are fairly unstable and difficult to isolate. Nevertheless, in many organic and biological reactions, it is well known that imines play an important role as intermediates. Thus, if the present reaction does involve the condensation of aromatic amines with reducing sugars, the acyclic form of the sugar will presumably be involved in the first step of the reaction, and it is predicted that the formation of the glycosylamine may proceed through a carbinolamine and an immonium ion $[\mathrm{R}-\mathrm{CH}=$ $\left.\mathrm{NHR}^{\prime}\right]{ }^{13)}$ Therefore, because of the instability of imines or Schiff bases, the formation of the glycosylamines can be expected to proceed also in aqueous solutions, as reported by Ikeda. ${ }^{3)}$

Next, we examined whether or not the UV absorption spectra, the chromatographic behavior, and the mode of hydrolysis of the authentic glycosylamine are consistent with those of the amine-carbohydrate reaction products as determined by HPLC analysis.

The hydrolytic behavior of authentic glycosylamine was observed by HPLC and UV spectroscopy, and the results agreed well with those obtained by diluting the equilibrated amine-carbohydrate mixture. Details of the kinetic studies will be published elsewhere. During the hydrolysis of $N$-PABA-dRib at $\mathrm{pH} \mathrm{7.0,} \mathrm{the} \mathrm{spectral} \mathrm{changes} \mathrm{were} \mathrm{similar} \mathrm{to} \mathrm{those}$ found on dissociation of the reaction product (Fig. 4). Figure 5 shows the spectral changes during $N$-p-carboxyphenyl-D-glucosylamine ( $N$-PABA-Glu) hydrolysis at $\mathrm{pH} 1.26$. Remarkable spectral changes were observed with a slight hypsochromic shift of the absorption maxima. The marked changes may arise from the protonation of the primary aromatic amine in such a strongly acidic region. As can be seen in Fig. 5, N-PABA-Glu was completely hydrolyzed to free PABA and D-glucose after $16 \mathrm{~h}$ under the conditions used. 


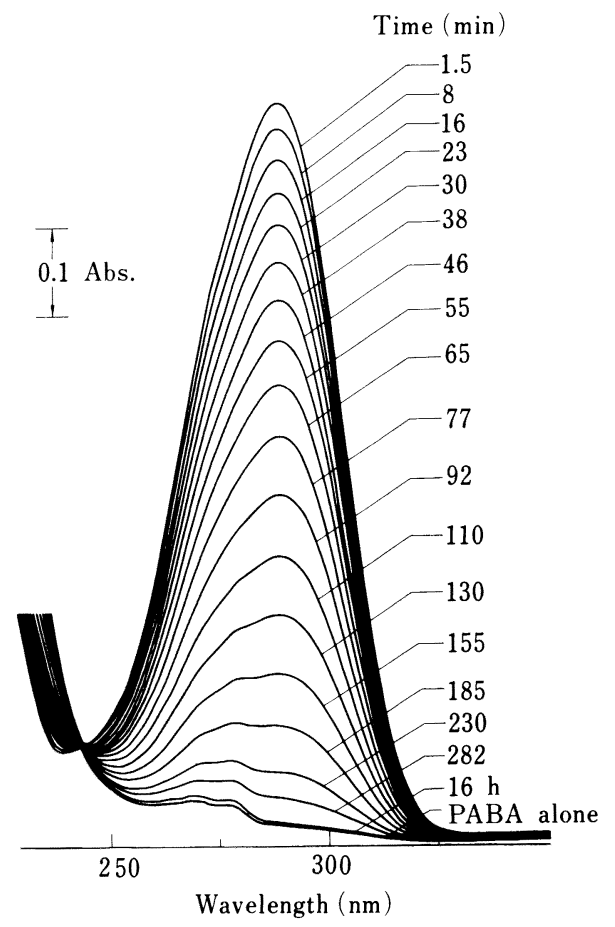

Fig. 5. UV Spectral Changes Accompanying the Hydrolysis of $N$-p-Carboxyphenyl-D-glucosylamine ( $N$-PABA-Glu) at $\mathrm{pH} 1.26$

Authentic $N$-PABA-Glu was dissolved in aqueous hydrochloric acid-potassium chloride solution $(\mathrm{pH}$ 1.26 , ionic strength 0.3 ), to give a final concentration of $3.20 \times 10^{-5} \mathrm{M}$.

The retention times of the authentic samples were in good agreement with those of the reaction products found in aqueous amine-carbohydrate mixtures. A chromatogram for the PABA-glucose system is shown in Fig. 6(A); the retention time of the PABA-glucose reaction product (peak $1, t_{\mathrm{R}} 2.4 \mathrm{~min}$ ) agreed well with that of authentic $N$-PABA-Glu (peak 3), though the nature of the minor peak just before peak 3 is unknown. Moreover, by using an HPLC apparatus equipped with a photodiode array detector, UV spectra of the three compounds (peaks 1-3) were recorded at the respective elution peaks during an HPLC run, as shown in Fig. 6(B). In addition to the similarity of the spectra of the reaction product and authentic glucosylamine over a wide wavelength region, the absorption maximum of the former $\left(\lambda_{\max }\right.$ $293 \mathrm{~nm}$ ) agreed well with that of the latter, and the bathochromic shift of $\lambda_{\max }$ by about $4 \mathrm{~nm}$ was also found, as anticipated from the dissociation profiles of the PABA-dRib and PABAglucose systems (see Figs. 4 and 5).

From the above results and discussion, the reaction products found chromatographically in aqueous solutions containing reducing sugars and drugs with a primary aromatic amine moiety can be identified as the glycosylamines. Therefore, the procainamide-glucose complex detected by HPLC $^{5-7)}$ seems not to be an association complex, but a chemical reaction product (a glucosylamine). In a mixture of procainamide and glucose, we also found a new peak in HPLC attributable to $N$-(D-glucosyl)procainamide.

In spite of the long-standing finding of the formation of glycosylamines by reaction between primary aromatic amines and reducing sugars in aqueous solution the reaction has not been well understood, as is clear from the recent reports. ${ }^{5-7)}$ It is worth noting that several $N$-(substituted phenyl)-D-glycosylamines have been synthesized and tested for ability to inhibit the growth of several malignant animal tumors ${ }^{14)}$ and the replication of cultured B 16 melanoma cells. ${ }^{15}$ ) Although in these studies the stability of the glycosylamines in the cell culture medium was not taken into account, some of these compounds were evaluated as new bioactive compounds independent of the parent amino compounds. It seems necessary to 


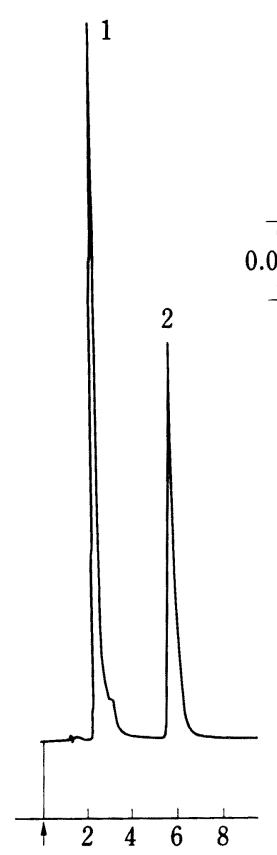

inj. Time $(\mathrm{min})$
(A)

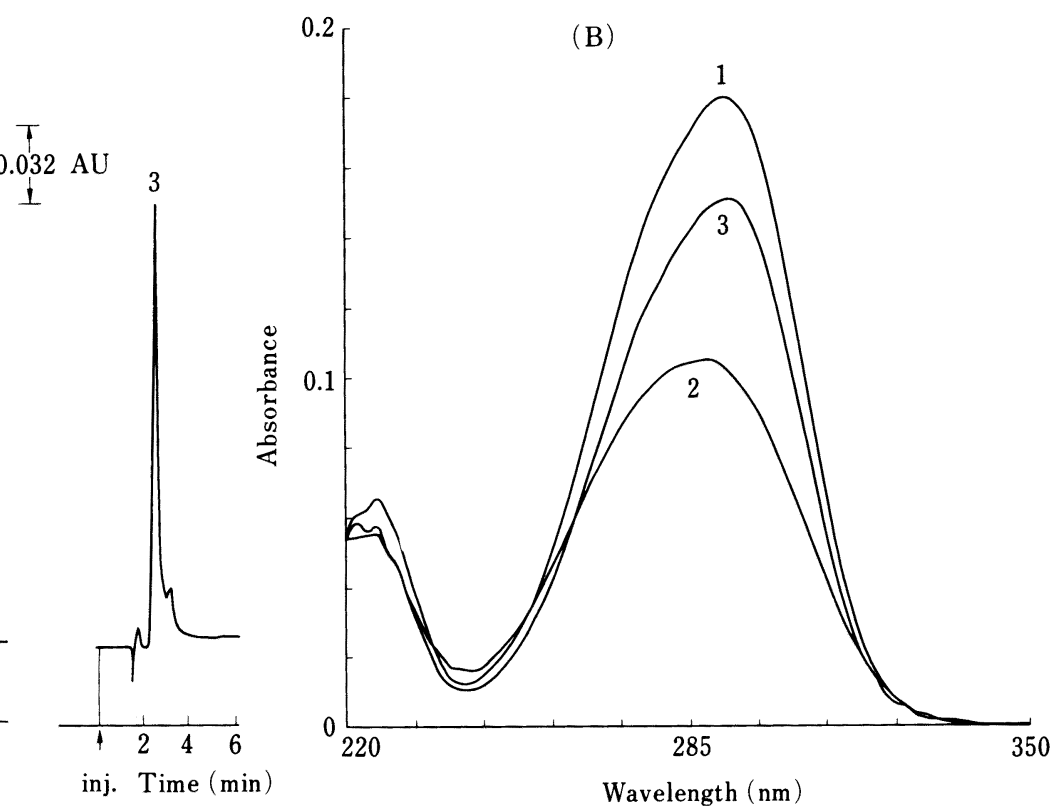

Fig. 6. HPLC Chromatograms and UV Absorption Spectra of PABA, PABAGlucose Reaction Product, and Authentic $N$-p-Carboxyphenyl-D-glucosylamine ( $N$-PABA-Glu), Obtained with a Photodiode Array Detector

(A) HPLC chromatograms; peaks $1-3$ denote PABA-glucose reaction product, free PABA, and authentic $N$-PABA-Glu, respectively.

(B) UV spectra of the above three compounds, recorded at the respective elution peaks during an HPLC run by using a photodiode array detector. Curves $1-3$ denote, PABAglucose reaction product, PABA and authentic $N$-PABA-Glu, respectively.

investigate in detail the formation and dissociation of glycosylamines, because the pharmaceutical and biological significance of these phenomena is still not sufficiently clear.

Acknowledgements The authors are grateful to Dr. T. Ibuka of the Faculty of Pharmaceutical Sciences, Kyoto University, for valuable advice and discussions and to Prof. M. Masui of the Faculty of Pharmaceutical Sciences, Osaka University, for HPLC analysis employing a photodiode array detector.

\section{References and Notes}

1) a) J. S. Cannell, Pharm. J., 167, 231 (1951); b) Idem, J. Pharm. Pharmacol., 3, 741 (1951).

2) T. Kaito, Bull. Natl. Inst. Hyg. Sci., 72, 99, 101 (1954).

3) K. Ikeda, Chem. Pharm. Bull., 5, 101 (1954).

4) a) S. L. Hem, E. J. Russo, S. M. Bahal, and R. S. Levi, J. Pharm. Sci., 62, 267 (1973); b) C. Larsen and H. Bundgard, Arch. Pharm. Chem., Sci. Ed., 6, 33 (1978); c) R. H. Barry, M. Weiss, J. B. Johnson, and E. DeRitter, J. Pharm. Sci., 71, 116 (1982).

5) H. L. Kirschenbaum, L. J. Lesko, R. W. Mendes, and G. P. Sesin, Am. J. Hosp. Pharm., 36, 1464 (1979).

6) D. M. Baaske, A. W. Malick, and J. E. Carter, Am. J. Hosp. Pharm., 37, 1050 (1980).

7) a) V. Das Gupta, J. Pharm. Sci., 71, 994 (1982); b) Idem, ibid., 72, 205 (1983).

8) The name "glycosylamine" is recommended as the general name for the compounds resulting from the condensation of aldoses and ketoses with ammonia and amines (Editorial Report on Nomenclature, J. Chem. Soc., 1952, 5108.

9) G. P. Ellis and J. Honeyman, Adv. Carbohydr. Chem., 10, 95 (1955).

10) A. N. Martin, J. Swarbrick, A. Cammarata, and A. H. C. Chun, "Physical Pharmacy," 2nd Ed., Lea \& Febiger, 
Philadelphia, 1969, p. 331.

11) W. Pigman and H. S. Isbell, Adv. Carbohydr. Chem., 23, 11 (1968).

12) L. Pauling, "The Nature of the Chemical Bond," 3rd Ed., Cornell University Press, New York, 1960, Chap. 7.

13) H. S. Isbell and H. L. Frush, J. Org. Chem., 23, 1309 (1958).

14) T. Kariya, H. Oka, T. Oda, M. Ikuzawa, T. Fuji, C. Yoshikumi, T. Hotta, and M. Yanagisawa, Curr. Chemother. Infect. Dis., Proc. Int. Congr. Chemother., 11 th, 2, 1950 (1980).

15) a) Lin Wang, C. A. Maniglia, A. L. Mella, and C. Sartorelli, J. Med. Chem., 26, 629 (1983); b) Idem, ibid., 26, $1323(1983)$ 